\title{
Temporary cardiac arrest in patient under robotically assisted total thyroidectomy causing carotid sinus hypersensitivity -A case report-
}

\author{
Hyun Sik Chung, Chong Min Park, Eun Sung Kim, Bo Gyoung Ghil, and Chul Soo Park \\ Department of Anesthesiology and Pain Medicine, College of Medicine, The Catholic University of Korea, Seoul, Korea
}

\begin{abstract}
Carotid sinus hypersensitivity (CSH) is an exaggerated response to carotid sinus baroreceptor stimulation. Bradycardia, hypotension, and syncope are common manifestations of CSH. A 31-year-old female patient was scheduled for a robotically assisted endoscopic total thyroidectomy. No problems occurred during anesthetic induction. Sudden cardiac arrest occurred near dissection of the diseased thyroid. However, while atropine was administered, the patient soon recovered to normal sinus rhythm. Subsequent bradycardia or hypotension was not followed until the end of surgery. (Korean J Anesthesiol 2010; 59: S137-S140)
\end{abstract}

Key Words: Cardiac arrest, Carotid sinus hypersensitivity, Robot, Thyroidectomy.

Patients who undergo surgery can face various complications resulting from medical or surgical causes. Among these complications, cardiac arrhythmia is a very serious cause that contributes to morbidity and mortality during the perioperative period [1]. Sinus bradycardia is known as one of the most common arrhythmias to occur during the intraoperative period and until postoperative 24 hours. Reported common causes for bradycardia include anesthetic agents, central neural blockade, and vagal reflex by surgical stimulus [2]. Compared to other causes, prediction of occurrence of the vagal reflex is difficult. Therefore, if procedures used during surgery involve the carotid sinus, innervated by the vagus nerve, the possibility of triggering arrhythmia or hypotension should be considered. Even local stimulation of the carotid sinus can stimulate an extreme reaction of the baroreceptor.

As we experienced a cardiac arrest during robotically assisted total thyroidectomy by local stimulation of the carotid sinus, we report this case along with other literary contemplations.

\section{Case Report}

A 31-year-old female patient who was diagnosed with thyroid cancer underwent robotically assisted total thyroidectomy. The patient was $157 \mathrm{~cm}$ tall and weighed $47 \mathrm{~kg}$. Other than

Received: February 25, 2010. Revised: 1st, March 12, 2010; 2nd, April 28, 2010. Accepted: May 7, 2010.

Corresponding author: Chul Soo Park, M.D., Department of Anesthesiology and Pain Medicine, College of Medicine, The Catholic University of Korea, 505, Banpo-dong, Seocho-gu, Seoul 137-040, Korea. Tel: 82-2-2258-2236, Fax: 82-2-537-1951, E-mail: p6c8s17@catholic.ac.kr

This was presented at the $86^{\text {th }}$ Annual Scientific Meeting of the Korean Society of Anesthesiologists, Nov 6-7, 2009, International Convention Center, Jeju, Korea.

(c) This is an open-access article distributed under the terms of the Creative Commons Attribution Non-Commercial License (http:// creativecommons.org/licenses/by-nc/3.0/), which permits unrestricted non-commercial use, distribution, and reproduction in any medium, provided the original work is properly cited. 
appendectomy 1 year earlier, she had no special past medical or surgical history. She had a history of allergy to shellfish and grogram. According to preoperative electrocardiogram, no abnormal findings were identified (normal regular sinus rhythm and heart rate 70 beats per minute). Therefore, additional tests in association with cardiac electrophysiology and carotid sinus hypersensitivity were not attempted before surgery. Results from blood chemistry tests, including serum electrolytes, were normal.

Premedication was not given preoperatively. After preoxygenation, 2\% propofol solution (Fresofol, Fresenius Kabi, Bad Homburg, Germany), $50 \mathrm{ml}$ in the Schinder model, and remifentanil (Ultiva, GlaxoSmithKline, Brentford, UK), which was diluted to $40 \mu \mathrm{g} / \mathrm{ml}$ in the Minto model, were intravenously administered for induction and maintenance using a target controlled infusion pump. During induction, target effect concentrations of propofol and remifentanil were adjusted to $5 \mu \mathrm{g} / \mathrm{ml}$ and $4 \mathrm{ng} / \mathrm{ml}$, respectively. Rocuronium $50 \mathrm{mg}$, was used to induce muscle relaxation for intubation, which was intraoperatively maintained using atracurium diluted to $5 \mathrm{mg}$ / $\mathrm{ml}$ by normal saline and continuously infused at a rate of $5 \mu \mathrm{g} /$ $\mathrm{kg} / \mathrm{min}$.

No problematic events occurred during induction or intubation. For maintenance of anesthesia, the target effect concentrations of propofol and remifentanil were $4 \mu \mathrm{g} / \mathrm{ml}$ and $3 \mathrm{ng} /$ $\mathrm{ml}$, respectively, and were adjusted by units of 0.5 , if needed. Mechanical ventilation was supported by nitrous oxide $2 \mathrm{~L} / \mathrm{min}$ and oxygen $2 \mathrm{~L} / \mathrm{min}$. Radial artery cannulation was performed for tight monitoring of vital signs during surgery.

The patient's neck was hyperextended and the left arm was elevated, stretched, and fixed in supine position for passage of endoscopy and surgical devices via an axillary approach. For insertion of robotic arms, the surgeon made a $3.5 \mathrm{~cm}$ sized perpendicular incision at the axillary area and dissected tissues under the platysma muscle from the upper border of the pectoralis major muscle to the inner border of the sternocleidomastoid muscle using a Harmonic Scalpel. For visibility of surgery, an extractor was inserted in the incised axillary area, and connected to an elevator for elevation of the dissected flap. Another $0.5 \mathrm{~cm}$ sized incision was made between the midpoint of the left clavicle and the left nipple for insertion of a camera in the robot arm for visualization of the surgical field. The robot was placed at the right side of the patient, and its three arms were inserted into the incised axillary area for surgery. To aid the surgeon, one assistant inserted an endoscopic device through the incised axillary area. To expose the thyroid gland, a total of four arms of the robot and one endoscopic device were used in dissection of the sternohyoid muscle from the inner border of sternocleidomastoid muscle.

The patient's vital signs were managed stably at the beginning of surgery. Systolic and diastolic blood pressure were maintained in a range of $100-120 \mathrm{mmHg}$ and $60-80$ $\mathrm{mmHg}$, respectively, and heart beat showed regular sinus rhythm in a range of 70-80 rates per minute. The surgeon dissected ipsilateral left thyroid tissue at the incision site, and then the contralateral side of the thyroid, except for the isthmus area. Around the time when the left thyroid area was almost dissected, the robotic arms crossed the left carotid bifurcation, and multiple manipulations in that area were broken. At that time, a sudden absence of electrical waves was detected on the electrocardiogram, arterial line, and pulse oximetry. Sinus bradycardia or hypotension in monitoring of vital signs was not preceded before the attack. As soon as we recognized cardiac arrest, we urged the surgeon to stop surgical manipulation and administered atropine $0.5 \mathrm{mg}$ intravenously. The patient's cardiac rhythm was restored to normal sinus rhythm in a short time (Fig. 1). Total length of cardiac arrest time was 5.8 seconds. During cardiac arrest, no electrical wave was observed in the electrocardiogram, right radial arterial blood pressure, and peripheral oxygen saturation monitoring device. After recovery to normal sinus rhythm, sinus tachycardia of $100-110$ rates per minute continuously was followed temporarily. However, no recurrence of cardiac arrest, sinus bradycardia, or hypotension was observed. Atracurium infusion was stopped near 30 minutes before the end of surgery and pyridostigmine and glycopyrrolate were administered for reverse neuromuscular block. After the patient's self-respiration recovery was confirmed, extubation was safely performed in the operating room.

After restoration of consciousness in the post anesthetic care unit (PACU), we inquired and investigated the patient's

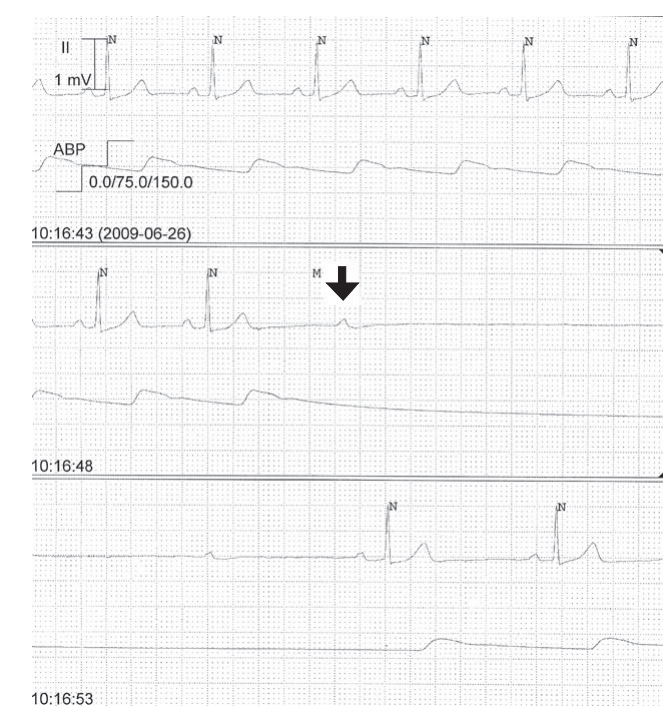

Fig. 1. Temporary cardiac arrest on electrocardiography (II) and right radial arterial pressure $(\mathrm{ABP})$ during surgery. 
past medical history again. However, she had no special problems, such as syncope, headache, or dizziness. Neurologic examination showed no abnormal findings at that time. The patient stayed in the PACU for one and a half hours without any problems, and then sent to the general ward. The patient was discharged four days after surgery with no special sequelae.

\section{Discussion}

Carotid sinus hypersensitivity (CHS) is an exaggerated response of the carotid sinus baroreceptor to local stimulation. According to Kim et al. [3] the incidence of intraoperative CSH was $28 \%$ in elderly patients, and $10 \%$ in young patients undergoing radical neck dissection. Therefore, tight monitoring of the patient's pulse rate and blood pressure is recommended in such surgery.

The carotid sinus is located on the internal carotid artery bifurcation and plays a central role in maintenance of homeostasis of blood pressure [4]. Afferent stimulus of the carotid sinus reflex is transmitted via myelinated or unmyelinated fibers of the glossopharyngeal and vagus nerves, and finally reaches the nuclei tractus solitarius and para median reticular nucleus in the brainstem. Efferent signals are relayed through sympathetic and vagus nerves to the heart and blood vessels, controlling heart rate and vasomotor tone. In the carotid sinus reflex, atrioventricular conduction delays, resulting in bradycardia and decreased sympathetic vascular tone, causing hypotension by enhancement of parasympathetics [5].

Although the exact mechanism and site of abnormal sensitivity is unknown, the exaggerated response may be due to changes in either part of the reflex arc or target organs. Local factors that exert pressure on the carotid sinus such as tumor, lymph node, and scars may lead to CSH $[6,7]$. CSH is clinically categorized into 3 types. The first is the cardioinhibitory type, which causes decreased heart rate, such as sinus bradycardia or arrest. This type occupies $70-75 \%$ of total CSH cases. The second is the vasodepressor type, which has no effect on heart rate, but depresses vasomotor tone; $5-10 \%$ of total CHS cases can be explained by this type. The third is the mixed type, which occurs in $20-25 \%$ of total CSH cases. Decreases in heart rate and vasomotor tone can be complicated $[4,5,8]$. In this case, the patient underwent cardiac arrest for 5.8 seconds; however, blood pressure did not change before or after the attack. Therefore, the cardioinhibitory type of CSH could be thought of as cause of cardiac arrest.

Carotid sinus massage (CSM) is essential for diagnosis or categorization of CSH type. The exact location of CSM is between the carotid pulse and the medial side of the sternocleidomastoid muscle from the level of thyroid cartilage to the angle of the mandible. Massage must be maximally performed for 5 seconds and stopped if cardiac arrest occurs for longer than 3 seconds. Similar to $\mathrm{CSH}$, results of a positive stress test can be categorized according to 3 types. Although CSM is associated with few complications, neurologic complications, coronary artery spasm, and ventricular arrhythmia can occur in less than $0.2 \%[9,10]$. In this case, because the patient showed no suspicious evidence of CSH prior to surgery, we were not able to apply CSM.

Depending on the frequency, severity, and consequences of each patient's symptoms, most patients with CSH do not require special treatment. Most patients can be managed well with education, lifestyle changes, expectancy, and routine follow-up. Patient treatment is different according to clinical types. For the cardioinhibitory type, anticholinergic drugs are recommended. According to Kenny et al. [11] atropine $0.7 \mathrm{mg}$ is the optimal dose for minimal complications and effective act on the muscarinic 2 receptor in the heart. For the vasodepressor type, ephedrine can be tried. For the mixed type, ephedrine alone is not enough, but can be effective when combined with a beta blocker. If bradycardia is severe or frequent, a temporary cardiac pacemaker should be considered such as dual chamber pacemakers (DDD, DVI, DDI). Surgical or radiological denervation of the carotid sinus nerve was previously a treatment option $[7,8]$. Sertraline and fluoxetine are reported to be effective in patients who do not respond to a dual chamber cardiac pacemaker. Alpha-1 agonist such as midodrine, or fludrocortisones have also been commented about CSH [12-14].

In this case, patient had no special past medical history, or abnormal findings on electrocardiogram and laboratory tests. During follow-up observation until the day after surgery, the patient showed no significant fluctuation of blood pressure in either supine or standing position. However, due to the unique aspects of robot assisted surgery, surgical procedures were more complicated than the usual endoscopic thyroidectomy; many instruments had to be concentrated in a small space in the neck. In general endoscopic thyroid surgery, endoscopic instruments have limitations in full mobilization; therefore, they can only move toward the distal end. In contrast, in robotic surgery, instruments from robotic arms can move more freely, which suggests a higher risk of carotid sinus stimulation [15]. Therefore, in this case, we thought that local mechanical stimulation by endoscopic instruments was the direct cause of temporary cardiac arrest during thyroidectomy. In consideration of some points, the patient went through self-recovery without special tests or treatment, and no more changes in vital signs followed; CSH from local stimulation of the carotid sinus could be a quite reasonable explanation for temporary cardiac arrest.

In conclusion, anesthesiologists should pay more attention to vital sign monitoring and thorough preparations for emergency 
for surgical procedures that involve manipulation of the carotid sinus and that are robotically assisted. If not contraindicated, atropine premedication may be helpful for prevention of a disastrous attack from CSH. Furthermore, risky surgical processes for stimulation of the carotid sinus should also be discussed with the surgeon before and during surgery.

\section{References}

1. Thompson A, Balser JR. Perioperative cardiac arrhythmias. Br J Anaesth 2004; 93: 86-94.

2. Ngamprasertwong P, Kositanurit I, Yokanit P, Lerdsirisopon S, Pulnitiporn A, Klanarong S. The Thai Anesthesia Incident Monitoring study (Thai AIMS): perioperative arrhythmia. J Med Assoc Thai 2009; 92: 342-50.

3. Kim TI, Lim HJ, Chang SH, Kim NS. Intraoperative carotid sinus hypersensitivity and postoperative complication of radical neck dissection retrospective study. Korean J Crit Care Med 1998; 13: 4954.

4. Thomas JE. Hyperactive carotid sinus reflex and carotid sinus syncope. Mayo Clin Proc 1969; 44: 127-39.

5. Muntz HR, Smith PG. Carotid sinus hypersensitivity: a cause of syncope in patients with tumors of the head and neck. Laryngoscope 1983; 93: 1290-3.

6. Babin RW, Panje WR. The incidence of vasovagal reflex activity during radical neck dissection. Laryngoscope 1980; 90: 1321-3.
7. Strasberg B, Sagie A, Erdman S, Kusniec J, Sclarovsky S, Agmon J. Carotid sinus hypersensitivity and the carotid sinus syndrome. Prog Cardiovasc Dis 1989; 31: 379-91.

8. Coplan NL, Schweitzer P. Carotid sinus hypersensitivity. Case report and review of the literature. Am J Med 1984; 77: 561-5.

9. Coplan NL. Carotid sinus hypersensitivity and syncope: cause/ effect or true/true/unrelated. Arch Intern Med 2006; 166: 491-2.

10. O'Shea D, Parry SW, Kenny RA. The Newcastle protocol for carotid sinus massage. J Am Geriatr Soc 2001; 49: 236-7.

11. Kenny RA, McIntosh SJ, Wynne H. Pattern of inhibition of parasympathetic activity in response to incremental bolus doses of atropine in carotid sinus hypersensitivity. Clin Auton Res 1994; 4: 63-6.

12. Moore A, Watts M, Sheehy T, Hartnett A, Clinch D, Lyons D. Treatment of vasodepressor carotid sinus syndrome with midodrine: a randomized, controlled pilot study. J Am Geriatr Soc 2005; 53: 114-8.

13. Grubb BP, Samoil D, Kosinski D, Temesy-Armos P, Akpunonu B. The use of serotonin reuptake inhibitors for the treatment of recurrent syncope due to carotid sinus hypersensitivity unresponsive to dual chamber cardiac pacing. Pacing Clin Electrophysiol 1994; 17: 1434-6.

14. Hussain RM, McIntosh SJ, Lawson J, Kenny RA. Fludrocortisone in the treatment of hypotensive disorders in the elderly. Heart 1996; 76: 507-9.

15. Yohannes P, Rotariu P, Pinto P, Smith AD, Lee BR. Comparison of robotic versus laparoscopic skills: is there a difference in the learning curve? Urology 2002; 60: 39-45. 